\title{
Virtual and on Site Vaccination Clinics of COVID-19. Will it be Applicable?
}

\author{
Sara $\mathrm{AR}^{2}$, Mohamed Raslan ${ }^{1,2}$, Eslam $\mathrm{MS}^{2}$ and Nagwa A Sabri ${ }^{1 *}$ \\ ${ }^{1}$ Department of Clinical Pharmacy, Faculty of Pharmacy, Ain Shams University, \\ Cairo, Egypt \\ ${ }^{2}$ Drug Research Centre, Cairo, Egypt \\ *Corresponding Author: Nagwa A Sabri, Department of Clinical Pharmacy, Faculty \\ of Pharmacy, Ain Shams University, Cairo, Egypt.
}

Received: December 03, 2021

Published: December 24, 2021

(C) All rights are reserved by Nagwa A Sabri., et al.

\begin{abstract}
Background: As a result of production and manufacturing of COVID-19 vaccines, mass vaccination and immunization will become critical part in controlling covid-19 spreading. The present vaccination facilities and strategies are not optimized for the most effective clinical and infection control methods.

Aim: Highlight the current vaccination approaches and to suggest some proposals to enhance the quality and increase the capacity of vaccination system.

Method: The current immunization procedures situation were addressed and proposals of some additional approaches for improving the process and to maintain infection control procedures to the best practices.

Conclusion: To acquire immunity in a short amount of time, vulnerable persons should be mass vaccinated. This will bring the COVID-19 epidemic under control, reducing the load on health-care institutions and the requirement for hospitalization dramatically. The suggested changes to the present vaccination system, as well as prior experiences, will assist in providing immunization services while protecting people's safety.
\end{abstract}

Keywords: Covid-19; Clinical Pharmacist; SARS-CoV-2; Immunization; Vaccination

\section{Introduction}

Coronaviruses have emerged as the primary pathogens in developing respiratory illness epidemics. They are members of a broad family of single-stranded RNA viruses that may cause illnesses ranging from a common cold to severe symptoms such as MERS and SARS [1]. The most prevalent clinical signs of COVID-19 are fever, cough, lethargy, malaise, and shortness of breath. Global worry about the virus has grown as a result of its high transmission potential, which may be accompanied by morbidity and fatality [2].
COVID-19 vaccines are being developed by a number of research and development teams across the world. As vaccines are likely to be developed and manufactured, mass vaccination and immunization will become an essential part of pandemic control. To bring the COVID-19 pandemic under control and significantly reduce hospitalization, morbidity, and death rates while reopening the economy, a large number of susceptible people should receive the vaccine in order to become immune to the virus in a short period of time. An example of more susceptible individuals are those patients with serious mental illness and depressive disorders [3]. Public health organizations have employed mass vaccination in the 
past, and it is being advocated as a possible solution for COVID-19 immunization [4].

Till the entire population get vaccinated, the major strategy to reduce the transmission of the virus in both health care settings and the population is strong infection control measures. Public awareness of dealing with highly contagious respiratory disorders is critical in reducing illness transmission, particularly in medium and low-income nations where health institutions have only a moderate capability to respond to outbreaks [5].

Several studies have found that wearing a face mask helps to reduce the spread of viral infections. Maintaining a 1.5-meter distance between individuals could help to limit infection spread and break the chain of covid-19 [6].

Adhering to personal protective measures like wearing face masks, face shields, gloves, alcohol-based disinfectants, social distancing, and monitoring client's temperature before they enter the vaccination clinic is essential. High numbers of vaccination requests and limited capacity of health care providers is considered the main obstacle in performing safe mass vaccination.

From this point we are aiming to highlight on some ideas that may provide safe vaccination service, with minimal crowded environment, good social distancing, and less COVID-19 infection risk.

\section{Current vaccination clinics situation}

The current vaccination clinics or centers are not optimized to the best clinical and infection control practices. By taking a look at the individual journey in the vaccination process procedures we can find the following:

- The subject register his vaccination request through the ministry of health covid-19 vaccination online platform.

- $\quad$ After that the subject receives via an SMS his request registration number.

- Within few days the subject will receive an SMS with his predetermined vaccination date, and place.

- On the due date, the subject goes to the predetermined vaccination center.

- In the administrative area, you must provide the coordinator with you national ID, and show him the SMS message.

- After that you will get your turn in the queue list.
- A medical investigation is performed for you during which you will be asked about some questions regarding vaccination inclusion and exclusion criteria, and you will be informed about vaccination type and you can get answered about what you need to know about the vaccine.

- After that you will sing and fingerprint on the informed consent.

- $\quad$ Then you get will the vaccine.

- $\quad$ After that, you will receive a copy of the informed consent, and the vaccination card in which you will find the second vaccination dose due date.

The aforementioned vaccination procedures in the current $\mathrm{CO}$ VID-19 pandemic situation have some drawbacks. The first one, some vaccination centers are not large enough to permit proper social distancing, and they are get crowded. The second one, the SMS message of the predetermined vaccination due is indicting the date and the place only, it should also indicate the exact time of vaccination so that everyone goes to the vaccine center on his predetermined date and time to avoid any chances of crowding.

The third drawback, in some cases some appointments are sent to the clients on the same day at a very early time e.g. at $12 \mathrm{am}, 6$ am during which the individual may be a sleep and upon walking up he will discover that he should go to take the vaccine. This will be inappropriate with many people to know suddenly that they should go today to get vaccinated.

A study demonstrates increased efficiency using a web- and mobile-based application to support mass vaccinations. The FluTracker and HANDI solution eased the burden on all groups involved with mass vaccinations through improved patient flow and throughput, reduced staffing requirements, and streamlined data collection. Impact on employees was reduced due to efficient preregistration and numerous mobile vaccination venues. From 2012 to 2015 , employee vaccination rates at non-mass clinic venues increased from $27 \%$ to $63 \%$ [7].

Proposed improvements and new ideas in the current vaccination system

- The current vaccination system can be optimized through early predetermination of vaccination date, (at least the client should be informed 24 hours before vaccination due). 
- On the other hand, vaccination time should be predetermined in order to make people go to get vaccinated at different day times to prevent place crowding and keeping proper social distancing.

- Home based vaccination service could be an option especially for those individuals above 60 years, and geriatrics with limited movements. Nurses can be sent to homes based on predetermined appointments for vaccinating old people.

- Mobile vaccination clinics (off-site) can aid in reducing the burden on traditional clinics (on-site). Mobile vaccination clinics should be supported with the required IT and wireless connectivity tools to enable online access to covid-19 vaccine requests database. Also, required fridges or freezers for vaccine preservation should be provided. The off-site clinics will provide an advantage for any pre-registered individual to give him the vaccine shot at any time once he or she passed on the clinic on the way.

- Drive-through facilities have previously been used successfully for disease immunization and testing during COVID-19. Despite their benefits and promising outcomes, drive-through facilities for mass vaccination can be challenging to plan design, and operate [8].

- $\quad$ The number and configuration of drive-through mass vaccination centers should be planned based on the demographic characteristics of the population, the availability of appropriate and accessible space and various staff to run the sites, traffic conditions, vaccine supply, and other logistical issues such as personal protective equipment $[8,9]$.

- Various designs and layouts for drive-through vaccination sites have previously been proposed and used. A drivethrough center typically has two to ten lanes with three to four stations per lane including triage and screening, registration, shot dispensing, and recovery and discharge. More dispensing lanes provide higher throughputs and can be more effective at preventing traffic overflow onto neighboring streets $[10,11]$.

- Previously, layout improvements for drive-through vaccination sites were performed that make this facility served between 2000 and 3000 vehicles and their passengers on a daily basis [12].

- Passengers receiving immunization are mostly protected against weather conditions in their vehicles; however, staff at drive-through vaccination sites may be less protected. So the design of drive-through vaccination facilities should consider weather conditions such as temperature, humidity, wind, and rain. In poor climate situations a McDonald's drive-through may be an alternate design option, with vaccination employees residing within a covered room or building and delivering immunization through an open door or window [13].

- $\quad$ Since clinical pharmacist interventions showed to enhance nurses' medication error awareness and found to be beneficial in lowering the rate and severity of medication errors among nurses working in hospitals [14]. Proper training for clinical pharmacist should be afforded to make them ready for contribution in mass vaccination campaigns, as they can alleviate the increased vaccination burden on nurses.

\section{Conclusion}

Mass vaccination of susceptible people should be performed in order to become immune to the virus in a short period of time. This will bring the covid-19 pandemic under control and significantly reduce burden on health care facilities and need for hospitalization. Besides, morbidity, and death rates will decrease. Under limited health care resources and high immunization requests maintaining protective measures is challenging. The proposed modifications in the current vaccination system and the previously applied experiences will aid in providing vaccination service while maintaining people safety.

\section{Bibliography}

1. Cascella M., et al. "Features, Evaluation and Treatment Coronavirus (COVID-19)". in StatPearls. Treasure Island, FL: StatPearls Publishing LLC (2020).

2. Huang C., et al. "Clinical features of patients infected with 2019 novel coronavirus in Wuhan, China”. Lancet 395.10223 (2020): 497-506.

3. Nagwa Ali Sabri., et al. "Depressive Disorders and Incidence of COVID-19: Is There a Correlation and Management Interference?" Psychological Disorders and Research 3.2 (2020): 3-7.

4. Asgary A., et al. "A Drive-through Simulation Tool for Mass Vaccination during COVID-19 Pandemic". Healthcare (Basel) 8.4 (2020): 469.

5. Li JY., et al. "The epidemic of 2019-novel-coronavirus (2019$\mathrm{nCoV}$ ) pneumonia and insights for emerging infectious diseases in the future". Microbes Infectious 22.2 (2020): 80-85.

Citation: Nagwa A Sabri., et al. "Virtual and on Site Vaccination Clinics of COVID-19. Will it be Applicable?". Acta Scientific Pharmaceutical Sciences 6.1

(2022): 141-144. 
6. Nagwa A Sabri., et al. "Impact of Applied Protective Measures of COVID-19 on Public Health". Acta Scientific Pharmaceutical Sciences 5.7 (2020): 63-72.

7. Melissa W McClung., et al. "Managing public health data: mobile applications and mass vaccination campaigns". Journal of the American Medical Informatics Association 25.4 (2018): 435-439.

8. Pate J. "Drive-Thru PODs: An Alternate Method of Mass Prophylaxis". Public Health Preparedness Summit; Washington, DC, USA (2007).

9. Carrico R. "Drive-Thru Flu Shots-A Model for Mass Immunization". Spectrum Press; Chicago, IL, USA (2002).

10. Knox D. In: Davenport, Dispensing Emergency Medications and Supplies. Fielding J.E., Teutsch S.M., editors. Oxford University Press; Oxford, UK: (2012): 243-253.

11. Pate J. "Drive-Thru PODs: An Alternate Method of Mass Prophylaxis". Public Health Preparedness Summit; Washington, DC, USA (2007).

12. Lee EK., et al. "Modeling and Optimizing the Public-Health Infrastructure for Emergency Response". Interfaces 39 (2009): 476-490.

13. Wessex Local Medical Committees. "Operational Aspects of Influenza Immunisation 2020/21". Wessex Local Medical Committees; Eastleigh, UK (2020).

14. Lobna S Mostafa., et al. "Evaluation of pharmacist-led educational interventions to reduce medication errors in emergency hospitals: a new insight into patient care". Journal of Public Health 42.1 (2020): 169-174.

\section{Assets from publication with us}

- Prompt Acknowledgement after receiving the article

- Thorough Double blinded peer review

- Rapid Publication

- Issue of Publication Certificate

- High visibility of your Published work

Website: www.actascientific.com/

Submit Article: www.actascientific.com/submission.php

Email us: editor@actascientific.com

Contact us: +919182824667

Citation: Nagwa A Sabri., et al. "Virtual and on Site Vaccination Clinics of COVID-19. Will it be Applicable?". Acta Scientific Pharmaceutical Sciences 6.1 (2022): 141-144. 